\title{
Ground-based observations for the validation of contrails and cirrus detection in satellite imagery
}

\author{
H. Mannstein ${ }^{1}$, A. Brömser ${ }^{1,2}$, and L. Bugliaro ${ }^{1}$ \\ ${ }^{1}$ Deutsches Zentrum für Luft- und Raumfahrt, Institut für Physik der Atmosphäre, Oberpfaffenhofen, Germany \\ ${ }^{2}$ UBIMET GmbH, 1200 Wien, Austria
}

Received: 23 November 2009 - Published in Atmos. Meas. Tech. Discuss.: 10 December 2009

Revised: 3 May 2010 - Accepted: 6 May 2010 - Published: 7 June 2010

\begin{abstract}
Contrails and additional cirrus clouds caused by air traffic have a potential warming effect due to their optical properties and their location in the upper troposphere. The effect of contrails is directly related to their coverage and optical properties, which both can be derived from satellite observations. However, considerable local and global uncertainties remain, as detection limits and efficiency are still unknown. A six months time series of the occurrence of high-level clouds and contrails was analysed visually using an all-sky camera situated at Oberpfaffenhofen (Southern Germany). It shows a contrail occurrence of $21 \%$ (fraction of time with visible contrails during one hour) which is nearly constant over daytime and a cirrus occurrence that increases from $27 \%$ in the morning to $48 \%$ in the evening, suggesting a possible influence of air traffic or, more probably, convective cloud formation. Furthermore, we compared selected all-sky camera images with data of the satellite instruments NOAA/AVHRR and MSG/SEVIRI. As expected, the fraction of contrails visible and detectable in satellite images depends strongly on their width. Of the contrails observed with the all-sky camera of $1-5 \mathrm{~km}$ width $60-65 \%$ are visually detectable in AVHRR data while only $17 \%$ are identified by an automated contrail detection algorithm (CDA). This means that the automated CDA detects approx. $28 \%$ of the contrails which are identified by visual inspection in AVHRR data alone. As far as SEVIRI is concerned, visual inspection yields $48 \%$ of the contrails of $1-5 \mathrm{~km}$ width, the CDA $19 \%$. That means $40 \%$ of all contrails visually identifiable in SEVIRI data are found by the automated algorithm. As far as cirrus detection using SEVIRI data is concerned, an auto-
\end{abstract}

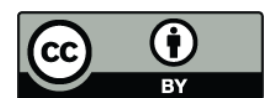

Correspondence to: H. Mannstein (hermann.mannstein@dlr.de) mated algorithm tends to overestimate cirrus occurrence but correctly measures cirrus changes during the day compared to visual inspection.

\section{Introduction}

Contrails (short for condensation trails) have been observed since 1915 (Schumann, 2005). The theory on the thermodynamic conditions causing their formation and decay was first described at the time of World War II (Schmidt, 1941). The strong increase in occurrence of contrails due to the strong increase of global air traffic has triggered intensive research in this field since the early 1990s, especially with respect to their present and future influence on global and regional climate (Meerkötter et al., 1999; Mannstein et al., 1999; Meyer et al., 2002; Marquart, 2003; Minnis et al., 2004).

Aircrafts add warm and humid exhaust air to the tropopause region. When the plume consisting of exhaust and entrained air cools, its relative humidity increases. If the ambient air is cold and moist enough, saturation with respect to liquid water is eventually reached. Soot particles from the combustion process and other condensation nuclei then start to accumulate water vapour and grow to little droplets which immediately freeze: the contrail is formed. The temperature and moisture limits are given by the Schmidt-Appleman Criterion (Schmidt, 1941; Appleman, 1953; Schumann, 1996). In warmer and moist surroundings contrails can also be initiated aerodynamically (Gierens et al., 2009; Kärcher et al., 2009). If the ambient air is supersaturated with respect to ice, the contrail can persist for several hours. Minnis et al. (1998) have observed life spans of more than $7 \mathrm{~h}$ for single contrails and $17 \mathrm{~h}$ for contrail clusters together with contrail widths of

Published by Copernicus Publications on behalf of the European Geosciences Union. 
$15 \mathrm{~km}$. Bakan et al. (1994) report contrail areas that could be tracked for more than two days. Nevertheless, a contrail usually loses its linear appearance at some point. After 1 or $2 \mathrm{~h}$ it can no longer be distinguished by its shape from a naturally formed cirrus cloud (Mannstein et al., 1999).

The most relevant parameters for the estimation of the climate impact of contrails are changes in cirrus cloud coverage and optical thickness, the resulting radiative forcing (RF), as well as possible effects due to changes in air composition and the influence on the hydrological cycle (Schumann, 2005). Like thin cirrus contrails reduce both, the incoming short wave radiation and the infrared radiation escaping into space (greenhouse effect). The former effect leads to an instantaneous surface cooling during daytime, the latter causes a warming mainly of the upper troposphere.

Cirrus cloud amount is on the one hand modified by contrails detectable due to their linear structure, on the other hand by additional contrail cirrus clouds that can only be detected indirectly by observing changes in cirrus cloud coverage. The global coverage of contrails is estimated to be in the range between $0.04 \%$ and $0.09 \%$ (Stuber and Forster, 2007), in Central Europe it amounts to $0.5 \pm 0.25 \%$ with regional maxima reaching 1.2\% (Mannstein et al., 1999). The coverage of aviation-induced cirrus is estimated to be 1.8 to 10 times higher than the one caused by contrails (Forster et al., 2007). According to Stordal et al. (2005) cirrus coverage in Europe increased by 1-2\% per decade, Zerefos et al. (2003) find a value of $1.8 \%$ for Europe. The optical thickness of contrails on global average lies between 0.15 and 0.25 (Minnis et al., 2005), Meyer et al. (2002) found a lower value of 0.1 for Europe. The radiative forcing caused by contrails is estimated to be between 3.5 and $17 \mathrm{~mW} \mathrm{~m}^{-2}$ based on air traffic conditions of 1992 (Schumann, 2005). In the current IPCC report (IPCC, 2007) it is estimated to be between 3 and $30 \mathrm{~mW} \mathrm{~m}^{-2}$, the best estimate being $10 \mathrm{~mW} \mathrm{~m}^{-2}$ for both the years 2000 and 2005 (Forster et al., 2007). According to Stordal et al. (2005) the RF of the additional cirrus ranges between 10 and $80 \mathrm{~mW} \mathrm{~m}^{-2}$ with a best estimate of $30 \mathrm{~mW} \mathrm{~m}^{-2}$. In a new assessment Lee et al. (2009) give an estimate of $12 \mathrm{~mW} \mathrm{~m}^{-2}$ for the linear contrails and 33 (1187) $\mathrm{mW} \mathrm{m}^{-2}$ for aviation induced cirrus. At present the influence of additional, aircraft-induced aerosol particles which might have an impact on cirrus coverage ("soot cirrus") on the Earth radiation budget cannot be quantified (Forster et al., 2007).

We present the analysis of six months of data from the all-sky camera "Wolkam" (this name stands for German "Wolkenkamera" meaning cloud camera), situated on the roof of the Institute of Atmospheric Physics located at the DLR Campus at Oberpfaffenhofen close to Munich. This is a region of heavy air traffic in the heart of Europe at a distance of approximately $50 \mathrm{~km}$ South-West of Munich Airport. From the nearly complete time series from April to September 2007 we derived monthly means and diurnal cycles of contrail and cirrus occurrence. Due to its high tem- poral and spatial resolution this dataset is ideally suited to validate space-borne algorithms for the detection of contrails and cirrus clouds. In particular, we focused on two satellite instruments: the polar orbiting NOAA/AVHRR and the geostationary MSG/SEVIRI. From data of these two sensors we derived contrail occurrence in two ways: (1) by applying the contrail detection algorithm developed by Mannstein et al. (1999), (2) by visual investigation of brightness temperatures, brightness temperature differences and false colour composites obtained from this satellite imagery. Thus, for each method we were able to estimate the contrail detection efficiency at the location of the all-sky camera as well as the minimum width that is needed for a contrail to be detected in NOAA/AVHRR and MSG/SEVIRI satellite data. Furthermore, cirrus detection from MSG/SEVIRI data was performed with the MeCiDA algorithm described in (Krebs et al., 2007) and compared to Wolkam data as well.

After a description of the all-sky camera and a short illustration of the satellite radiometers' main characteristics in Sect. 2, the visual inspection methods and the algorithms used as well as the intercomparison details are explained in Sect. 3. Section 4 presents the monthly time series and the diurnal cycles of cloud occurrences extracted from the allsky camera as well as the resulting contrail width distribution. The intercomparisons between Wolkam and AVHRR or SEVIRI are shown in Sects. 5 and 6 respectively, together with the estimation of the contrail detection efficiency and of the MeCiDA performance. Finally, conclusions are drawn in Sect. 7.

\section{Instruments}

\subsection{The Wolkam all-sky camera}

For ground-based contrail observations all-sky cameras are suitable (Sassen, 1997) because they are capable of covering almost the whole visible sky by the use of fisheye lenses and offer high temporal resolution (several images per minute). The Wolkam camera houses a CCD sensor providing colour images having a size of $2040 \times 2040$ pixels (32 MB in tiff format). The all-sky camera holds a 17 to $28 \mathrm{~mm}$ fisheye zoom objective yielding images with an opening angle of $90^{\circ}$ to $180^{\circ}$. In this study the minimum focal length is used resulting in the maximum viewing angle of $180^{\circ}$. These images cover all the visible sky except some areas close to the horizon, which is due to the square shape of the CCD chip. A neutral optical filter is used to damp direct sunlight. The optics and electronics are placed in a waterproof housing, the objective is located in the centre of an acrylic dome. Exposure time is automatically adapted to the prevailing light conditions by averaging the brightness of the whole image area of the proceeding images and adjusting it to a target value, shutter speeds in the range of $25 \mathrm{~ms}$ to $130 \mathrm{~s}$ are possible. 

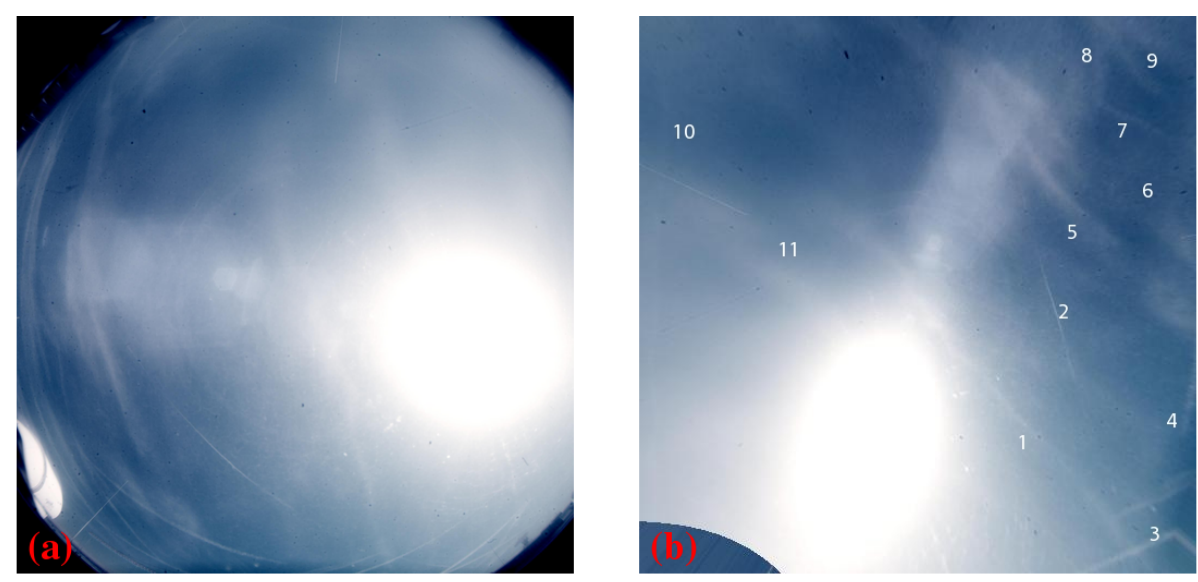

Fig. 1. Contrail scene of 16 April 2007, 12:02 UTC, as recorded by the Wolkam camera. (a) The original picture taken by the Wolkam all-sky camera. (b) The Wolkam picture after it has been deskewed, mapped on a plane, and aligned to the cardinal points. Here, 11 contrails could be identified and marked with numbers from 1 to 11 .

Table 1. Summary of the Wolkam, AVHRR and SEVIRI datasets.

\begin{tabular}{|c|c|}
\hline $\begin{array}{l}\text { Sensor, algorithm } \\
\text { and/or application }\end{array}$ & Dataset description \\
\hline Wolkam & April-September 2007, for a total of 2549.1 evaluable hours \\
\hline CDA on AVHRR & $\begin{array}{l}\text { 600-700 overpasses over Oberpfaffenhofen } 23 \text { March 2007-30 September 2007, with } 49 \text { over- } \\
\text { passes containing } 96 \text { Wolkam contrails }\end{array}$ \\
\hline CDA on SEVIRI (1) & hh:56 UTC MSG-2 slots of May 2007 (hh=00...23), for a total of 79 Wolkam contrails \\
\hline CDA on SEVIRI (2) & 2797 MSG-2 slots of May 2007, for a total of 299 contrails \\
\hline MeCiDA (SEVIRI) & All MSG-2 slots $(\approx 17500)$ April-September 2007 \\
\hline Wolkam (contrail width) & 175 contrails \\
\hline AVHRR (contrail width) & 96 contrails \\
\hline SEVIRI (contrail width) & 79 contrails \\
\hline
\end{tabular}

Routinely, the image is compressed to a $512 \times 512$ pixels jpg file of about $50 \mathrm{kB}$ size, and the original tiff file is deleted. This compression considerably reduces the storage effort and makes the visual evaluation of long time series of cloud occurrences possible (see below) without affecting their representativeness. Approximately six such jpgs are obtained every minute during daylight time, usually this process proceeds automatically without interruption. If an all-sky camera image is gathered within $30 \mathrm{~s}$ of a NOAA satellite overflight time, the original high-resolution tiff files are kept. An example with many contrails is shown in Fig. 1a.

All the images of the Wolkam camera are considerably distorted because of the wide angle optics. We determined the properties of the fisheye lens and sensor by comparing the moon position to the images taken in the first months of camera operation. It turned out that a spherical mapping with corrections for the zenith position, the radius of the sphere, and the azimuth direction within the image was able to reproduce the calculated moon positions with an error smaller than one degree, sufficient for our purposes. Assuming a constant altitude of $10000 \mathrm{~m}$ above ground for the contrails, the usual cruise altitude, the images are mapped onto an equidistant, north-south and east-west aligned grid with a resolution of $75 \mathrm{~m}$ per pixel covering approximately a $38 \mathrm{~km} \times 38 \mathrm{~km}$ square centred at Oberpfaffenhofen. This way, at least all parts of the sky with a zenith angle smaller than $62^{\circ}$ are displayed. A comparison of an unmapped and a mapped image is shown in Fig. 1a and b. In addition, Fig. 1b shows the 11 contrails that could be observed at that time. They are marked with small numbers from 1 to 11 .

The all-sky camera worked with only short interruptions from April to September 2007 for a total of evaluable $2549.1 \mathrm{~h}$ (see Table 1). This time series was inspected visually since an automatic image interpretation scheme was not feasible due to artefacts like blooming of a significant part of the image caused by the sun, improper focusing or reflections within the optical system, or morning dew on the acrylic dome.

This visual inspection was performed by a single individual to guarantee consistency of the resulting dataset. However, to exclude gross subjective misinterpretations of the camera images, a test has been conducted where four 
observers investigated a five day subset of the Wolkam data. This test proved that a certain amount of subjectiveness is present, but a satisfactory agreement was found.

\subsection{NOAA/AVHRR and MSG/SEVIRI}

The SEVIRI radiometer (Schmetz et al., 2002) of the geostationary MSG-2 (MET-9) satellite, situated at $0^{\circ}$ E longitude, provides an image of the whole visible surface of the Earth in 12 spectral channels every $15 \mathrm{~min}$. Its spatial resolution at the subsatellite point amounts to $3 \mathrm{~km}$ in all but one channel, the HRV (High Resolution Visible) channel, which has a spatial resolution of $1 \mathrm{~km}$.

The period of revolution of the polar-orbiting sunsynchronous NOAA satellites is about $102 \mathrm{~min}$, their altitude being around $850 \mathrm{~km}$. The AVHRR/3 (Goodrum et al., 2003) radiometer onboard features six spectral channels, a nadir spatial resolution of $1.1 \mathrm{~km}$ and a swath width of about $2500 \mathrm{~km}$. Data from NOAA12, NOAA14, NOAA17, and NOAA18 acquired at the German Remote Sensing Data Centre of DLR in Oberpfaffenhofen, Germany, was used. At the times of 49 NOAA overpasses (19 of NOAA12, 0 of NOAA14, 11 of NOAA17 and 19 of NOAA18) contrails have been observed in the camera images. The total number of AVHRR overpasses over Oberpfaffenhofen during the investigated time period amounted to 600-700.

\section{Methods}

\subsection{Visual evaluation of Wolkam data}

Every image of the all-sky camera has been classified according to five basic categories:

1. Low- and medium-level clouds: The visibility of the cirrus and therefore the contrail level is blocked or considerably limited by low- and medium-level clouds. According to experience the sky is classified in this category if more than 5 to 6 oktas of the sky is covered with these clouds.

2. Contrails without cirrus: There are contrails but no natural cirrus clouds visible in the camera's field of view. "Natural cirrus" means that a cloud is visually not classified as a contrail, which does not exclude any formation initiated by contrails. The presence of cirrus and spatially clearly separated contrails within one image belongs to this category and to category 4 at the same time (only few rare cases).

3. Contrails and cirrus: Contrails and cirrus clouds which are not clearly separated are visible.

4. Cirrus without contrails: Natural cirrus clouds but no contrails are visible (the rare simultaneous occurrence of cirrus and spatially clearly separated contrails belongs to this category and to category 2 at the same time). Again, natural cirrus means that it cannot be visually classified as a contrail.

5. Cirrus level visible, neither cirrus nor contrails: The images which do not belong to any other category, meaning the sky is devoid of clouds or there are lowor medium-level clouds covering less than about 5 oktas of the sky.

In order to obtain the time fraction of the appearance of contrails or cirrus (i.e. relative frequency of occurrence), three combined categories were added:

6. All contrails: Sum of "Contrails without cirrus" and "Contrails and cirrus".

7. All cirrus: Sum of "Contrails and cirrus" and "Cirrus without contrails".

8. All ice clouds: Sum of "All cirrus" and "Contrails without cirrus" (same as sum of "All contrails" and "Cirrus without contrails").

It has to be emphasised that the use of the illustrated procedure does not determine the cloud amount, the classification in the categories is always a binary decision ("yes" or "no") for each image.

The time of first and last occurrence of a cloud category were recorded. The accuracy of these time records is limited by several factors: slowly changing cloud structures (e.g. gradual transformation from cirrostratus into altostratus), broken cloud layers, or the non-consideration of clouds appearing close to the horizon. Thus, we assume an accuracy of $5 \mathrm{~min}$ in the error budget except for short-lived contrails.

Only contrails were considered that could be identified in the images for at least $3 \mathrm{~min}$. The consideration of all the contrails would have increased the number of points in time to be recorded considerably without clear changes in relative frequencies of the categories. Furthermore, short-lived and, at the same time, narrow contrails are hardly detectable in satellite imagery anyway and their impact on the large scale net radiation is considered to be negligible.

Finally, hourly sums of these cloud categories were computed. The frequencies were calculated as the fraction of all daylight hours (without times of data loss) as well as in relation to the period of visible cirrus level.

Our observations are not complete due to data loss, morning dew and shielding of the upper sky by lower clouds. Thus the sampling error, i.e. the uncertainty caused by the consideration of a limited sample of independent observations instead of the whole population has to be taken into consideration. The sampling error is defined as $\sigma_{n}=\sigma / \sqrt{n}$. Here the standard deviation $\sigma$ is 0.5 as only binary decisions occur. $n$ denotes the size of the sample, which was fixed by determining the amount of uncorrelated Wolkam images. It was 

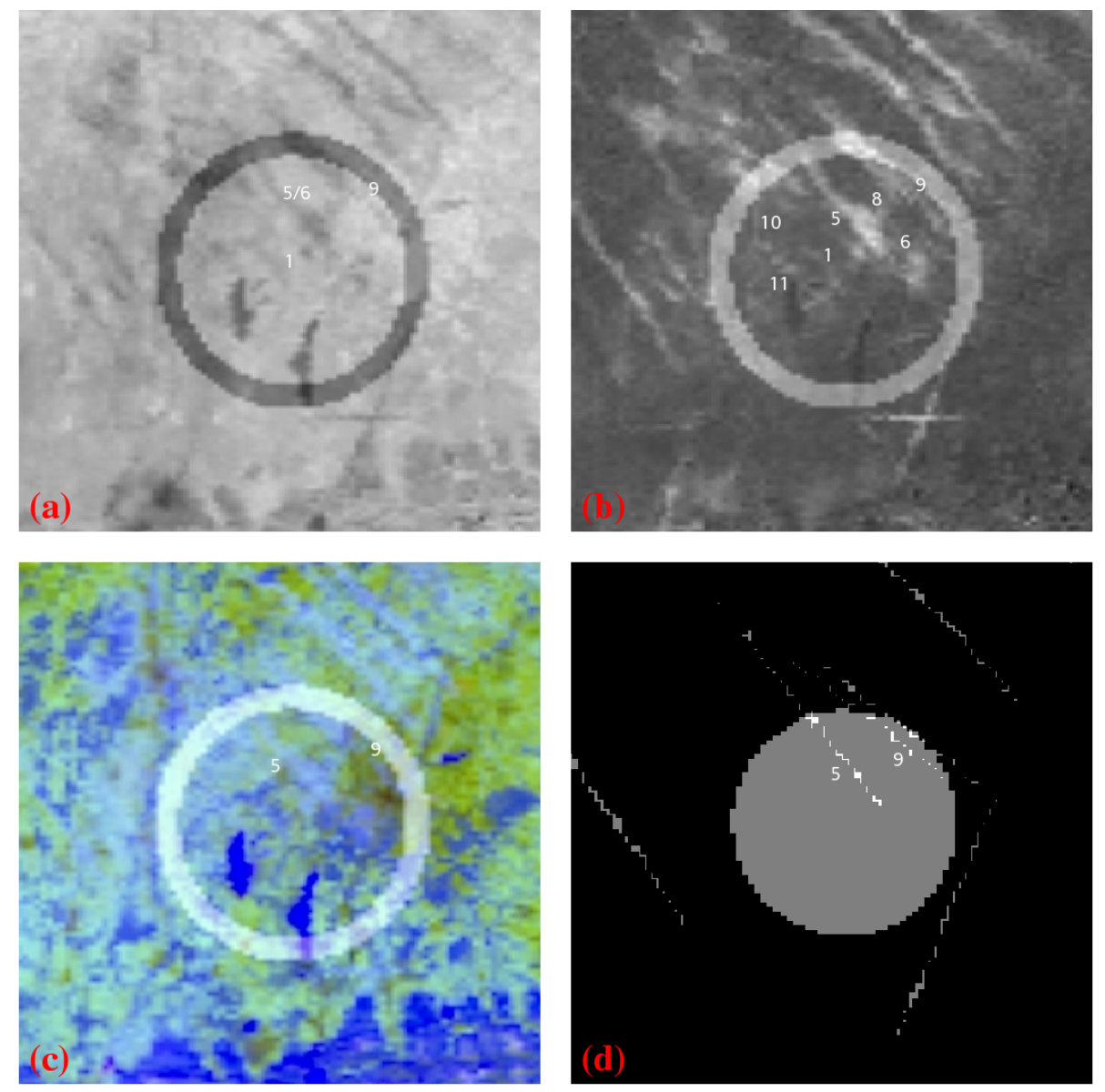

Fig. 2. Contrail scene of 16 April 2007, 12:02 UTC, as observed by the AVHHR instrument. All the image types used in this study are shown together with the result of the CDA. The corresponding Wolkam pictures are shown in Fig. 1. The Wolkam field of view is marked in all satellite pictures with a circle. (a) AVHRR channel 5 brightness temperatures. (b) AVHRR brightness temperature differences between channel 4 and channel 5. (c) AVHRR false colour composite. (d) Contrails detected by CDA: in grey outside of the Wolkam field of view, in white inside. The small numbers inside every panel indicate which contrails from Fig. $1 \mathrm{~b}$ could be detected in the corresponding AVHRR image.

assumed that after $20 \mathrm{~min}$ for low- and medium-level cloud situations and after $30 \mathrm{~min}$ for high-level clouds the pictures are no longer correlated. These time spans were estimated by visual inspection of approximately one dozen cloud scenes. The size $n$ of the sample is identified by calculating the number of uncorrelated Wolkam scenes using the whole measurement period within the particular time span, e.g. a month or a certain hour. If the sampling error exceeded $5 \%$ this data was not considered.

\subsection{AVHRR vs. Wolkam}

In the Wolkam images corresponding to the AVHRR overpass times 96 contrails could be visually detected between 23 March 2007 and 30 September 2007 (the time interval was slightly extended backwards in time in order to increase the number of contrails, see Table 1). AVHRR overpasses for which associated Wolkam images showed neither contrails nor cirrus were disregarded. The automated contrail detection algorithm (CDA) developed by Mannstein et al. (1999) was applied to the AVHRR data. The CDA uses a pattern recognition technique and an object classification scheme to differentiate line-shaped contrails from more fuzzy cirrus clouds. The algorithm is based on data from the $11 \mu \mathrm{m}$ and $12 \mu \mathrm{m}$ thermal infrared channels. It has been used in several studies (Meyer et al., 2002, 2007; Minnis et al., 2005) to derive the coverage by linear contrails. An overview article on the results of the CDA is in preparation.

An interesting but atypical case is presented in Fig. 2. Shown is the NOAA overpass on 16 April 2007 at 12:02 UTC corresponding to the Wolkam image of Fig. 1. It is atypical in the sense that 11 contrails could be observed at the same time. Usually only single contrails were found over Oberpfaffenhofen at the time of a NOAA overpass. In order to 
compare the AVHRR data to the Wolkam images the satellite data is mapped to a (roughly) equidistant cylindrical grid covering approximately $120 \mathrm{~km} \times 120 \mathrm{~km}$ centred at Oberpfaffenhofen, the location of the Wolkam camera. This larger surrounding area extending the Wolkam image's coverage facilitates the differentiation between contrails and natural cirrus. The Wolkam's field of view of $25 \mathrm{~km}$ radius is indicated in the images.

Figure 2 shows the four different AVHRR datasets used in order to visually detect contrails. In the channel 5 brightness temperatures contrails appear as dark, linear structures embedded in a brighter (warmer) background (Fig. 2a). Figure $2 \mathrm{~b}$ shows brightness temperature differences between AVHRR channel 4 and channel 5 (i.e. channel 4 minus channel 5) where contrails appear as bright lines contrasting a darker background. Figure $2 \mathrm{c}$ is a false colour composite using AVHRR channel 1,2 and 5 for the primary red, green and blue colours with contrails as light blue structures on top of an inhomogeneous background. Finally, Fig. 2d contains the CDA results for this overpass: black characterises non contrail pixels while grey pixels represent contrails outside and white pixels contrails inside the Wolkam field of view. CDA automated detections were considered successful if a contrail was detected within the $25 \mathrm{~km}$ circle that could be visually confirmed using the all-sky image (Fig. 1b).

All contrails that could be identified in one of the given AVHRR images are marked with a small number that refers to the related numbers in Fig. 1b. Especially the young and therefore thin contrails are emphasised in the brightness temperature difference image (Inoue, 1985). In some cases contrails become visually detectable this way which are not visible in the Wolkam images. In contrast, some aged wider contrails are visible in the Wolkam images but not in the temperature difference image. This brightness temperature difference is used as a thin cirrus test in remote sensing of clouds (Inoue, 1985). For transparent ice clouds this brightness temperature difference increases with decreasing particle size.

Summarising the example of Fig. 2, of the 11 numbered contrails visible in the all-sky camera image (Fig. 1b) 3 contrails are detectable in channel 5 temperatures, 7 in the temperature difference image, and 2 in both the false colour composite and the CDA result.

\subsection{SEVIRI vs. Wolkam}

The CDA originally developed for AVHRR has recently been adapted to MSG/SEVIRI and was applied to the 2797 MSG2 slots of May 2007 (see Table 1), a month with frequent occurrence of contrails. The necessary visual analysis of the satellite data is very time consuming as the temporal coverage of MSG-2/SEVIRI leads to a number of datasets within one month which is already four times higher than the number of AVHRR overpasses during the whole period $(\approx 2800$ datasets compared to $\approx 700$ ). This dataset could be used to determine contrail occurrence to compare to the Wolkam time series of the corresponding "All contrails" class.

For an additional CDA validation on SEVIRI, a visual analysis was performed analougous to that for AVHRR (Sect. 3.2). Wolkam images were compared to SEVIRI brightness temperature differences of channels 7 and 10 $(12.0 \mu \mathrm{m}$ minus $8.7 \mu \mathrm{m})$, SEVIRI false colour composites (using a channel combination including the high resolution SEVIRI channel 12 to increase spatial resolution: $1+12$ and $2+12$ for the primary colours red and green, and 10 for the blue component), and the output of the CDA algorithm on SEVIRI. As it has been shown in Krebs et al. (2007) the $12.0 \mu \mathrm{m}-8.7 \mu \mathrm{m}$ brightness temperature difference is an even better indicator for thin cirrus composed by small particles than the $12.0 \mu \mathrm{m}-10.8 \mu \mathrm{m}$ difference inherited from the AVHRR instrument.

From the SEVIRI data we selected an area equivalent to the one used with AVHRR. An area of $48 \times 24$ pixels centered at Oberpfaffenhofen was used as basis for the visual inspection. To facilitate the investigation, this area was enlarged and the region observed by Wolkam was marked in the images. As the deviations from the perfect north-south alignment are small in this region and an automated comparison of the images was not intended, a full mapping including a parallax correction was not done in this SEVIRI case. The situations showing contrails in the SEVIRI image but not in the all-sky camera image were neglected. Furthermore, this visual analysis was restricted to the SEVIRI data at minute 56 of each hour (see Table 1). Thus, multiple consideration of the same contrail was avoided as it typically takes more than 15 but less than 60 min to traverse the Wolkam's field of view. In the corresponding Wolkam dataset 79 contrails could be identified.

In addition, the Meteosat Second Generation Cirrus Detection Algorithm (MeCiDA) (Krebs et al., 2007) was applied to SEVIRI to determine the occurrence of cirrus clouds in the all-sky camera's field of view with high temporal resolution (15 min) during the full six month period (see Table 1). MeCiDA decides whether a SEVIRI pixel is cirrus covered by means of several threshold and morphological tests based on the thermal channels of SEVIRI. MeCiDA is a combination of six tests, each exploiting different channel combinations. In MeCiDA thin cirrus tests, based on brightness temperature differences involving mainly the atmospheric window channels, are combined with morphological tests, involving the two SEVIRI water vapour channels, in order to assure that the observed clouds are located in the upper troposphere. Further brightness temperature or brightness temperature difference tests are applied to detect thicker cirrus clouds as well as vertically extended clouds that are composed of ice particles in their upper part.

Since it turned out that for some reason almost always single pixels were classified as cloudy by MeCiDA inside the area of interest, we decided to build our analysis on a smaller area of $9 \times 9$ pixels around the all-sky camera: The larger 

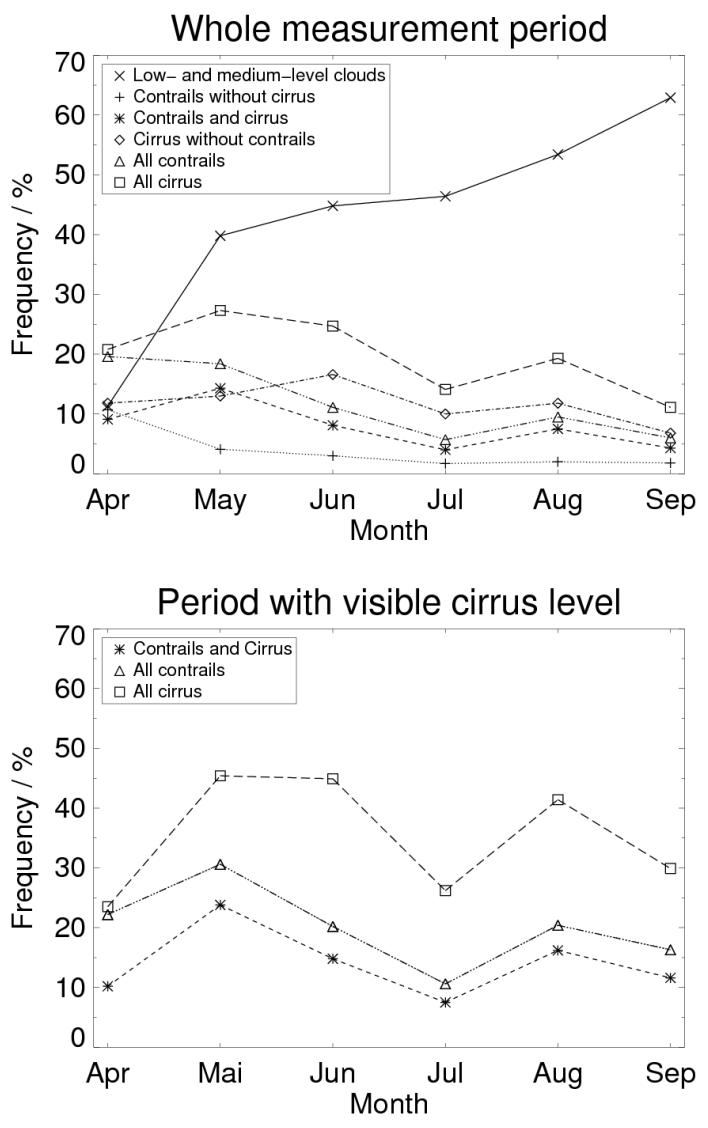

Fig. 3. Monthly mean values from the visual classification of ground based images. Top: Frequency of the occurrence of the categories "Low- and medium-level clouds" $(\times)$, "Contrails without cirrus" (+), "Contrails and cirrus" (*), "Cirrus without contrails" $(\diamond)$, "All contrails" $(\triangle)$, and "All cirrus" $(\square)$ w.r.t. the full measurement period. Bottom: Frequency of occurrence of the categories "Contrails and cirrus" $(*)$, "All contrails" $(\triangle)$ and "All cirrus" $(\square)$ when the cirrus level was visible.

$48 \times 24$ pixel area was first inspected to better identify cirrus structures, but the final decision about cirrus cloud occurrence was based upon the smaller region alone. The results of MeCiDA for the 81 SEVIRI low resolution pixels corresponding to the area covered by the all-sky camera observations were used in two ways: (1) in analogy to the analysis of the Wolkam time series, cirrus clouds and contrails (which are indistinguishable for MeCiDA) were identified if at least one of the pixels was classified as covered with cirrus; (2) cirrus cloud coverage was calculated as the fraction of the 81 SEVIRI pixels corresponding to the all-sky images where cirrus clouds were detected by MeCiDA. In addition, visual analysis of SEVIRI false colour composites and brightness temperatures were performed and the relative frequencies of cirrus cloud occurrence was determined. To allow for the comparison of the SEVIRI and Wolkam datasets, the SEVIRI products were aggregated for each hour of the day.
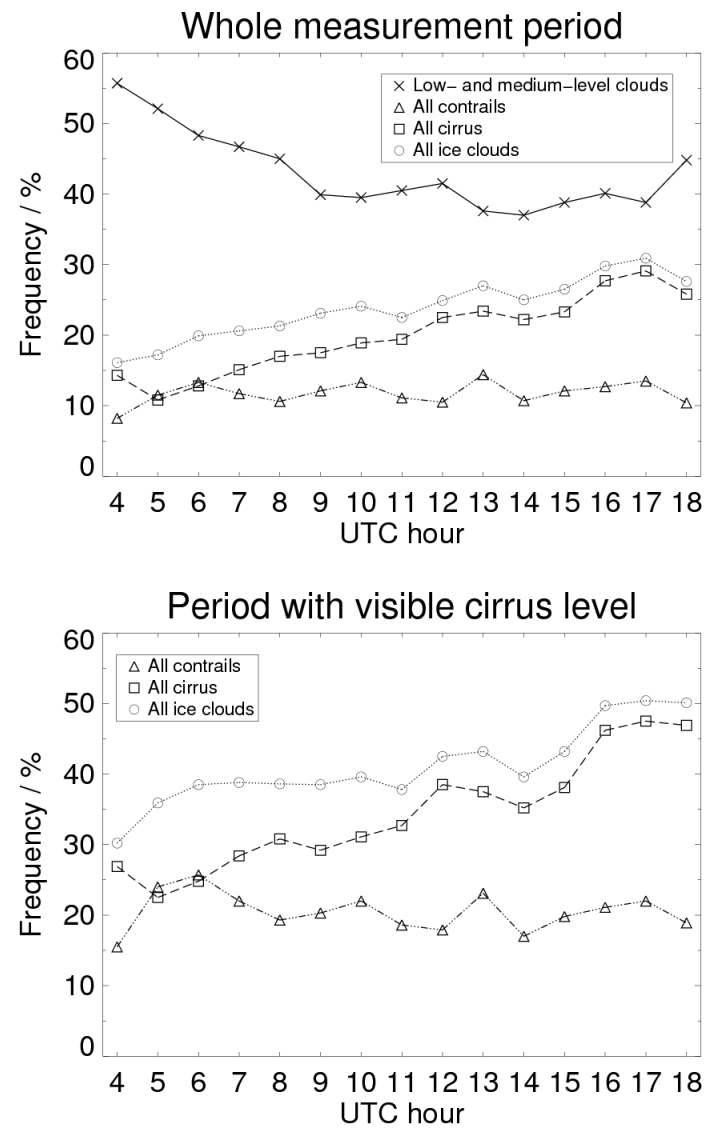

Fig. 4. Diurnal cycles of cloud classes obtained from the visual classification of ground based images. Top: Frequency of occurrence of the categories "Low- and medium-level clouds" $(x)$, "All contrails" $(\triangle)$, "All cirrus" $(\square)$ and "All ice clouds" (o), w.r.t. the full measurement period. Bottom: Frequency of occurrence of the categories "All contrails" $(\triangle)$, "All cirrus" $(\square)$ and "All ice clouds" (०), w.r.t. the time when the cirrus level was visible from the ground.

\subsection{Contrail width}

For the full dataset of 175 contrails from visual analysis of the all-sky camera images, including the very short-lived ones, the width was determined (see Sect. 4.3). This is a prerequisite for the estimation of the minimum width detectable in AVHRR or SEVIRI images (Sect. 5 and 6).

The assessment of the contrail width in the all-sky camera was based on the assumption that one pixel of the mapped camera image corresponds to $75 \mathrm{~m} \times 75 \mathrm{~m}$ when the contrails are situated $10 \mathrm{~km}$ above the ground. The deviation from the real flight level is smaller than $2 \mathrm{~km}$ in more than $90 \%$ of all cases according to a diagram published by Fichter et al. (2005). This results in a pixel width error smaller than $15 \mathrm{~m}$ for $90 \%$ of all cases. Assuming a starting width of $60 \mathrm{~m}$ and a rate of broadening of $50 \mathrm{~m} \mathrm{~min}^{-1}$ (values taken from Mannstein et al., 1999), this width is already reached after 
$1 \mathrm{~min}$. This implies the possibility of observing almost all contrails using an all-sky camera, especially if difference images of two consecutive shots separated in time by several seconds are used to accentuate thin contrails.

Subsequently, the contrails were divided into five classes according to their width to investigate the relationship between width and detection efficiency (for this the efficiency of the all-sky camera is set to $100 \%$ ). The classes comprise widths $<0.5 \mathrm{~km}, 0.5-0.9 \mathrm{~km}, 1.0-1.9 \mathrm{~km}, 2.0-4.9 \mathrm{~km}$, and $\geq 5 \mathrm{~km}$.

Finally, for AVHRR the brightness temperature difference between each contrail, detectable in both the all-sky camera and the satellite image, and its surrounding area was determined. This difference was, e.g., used to estimate the contrail optical thickness by Meyer et al. (2002).

\section{Ground-based observations of cirrus and contrails}

\subsection{Monthly variations of cirrus and contrail occurrence}

The monthly distribution of the categories $1-4,6,7$ (Sect. 3.1) frequencies relative to the whole measurement period is presented in Fig. 3 (top). The frequency of the "All contrails" occurrence fluctuates between 6\% in July 2007 and almost $20 \%$ in April 2007 with a mean value of $12 \%$, the one of "All cirrus" features a maximum of $27 \%$ in May and a minimum of $11 \%$ in September (mean value $20 \%$ ). A positive correlation of both classes is suggested by the graphics. The expected negative correlation to "Low- and mediumlevel clouds" is also observable. The remarkable small frequency of the last-mentioned category in April 2007 (11\%) is due to the unusually sparse occurrence of cloudy conditions in this month. Its continuous increase from April to September is related to the general weather situation peculiar to that particular year. While April 2007 was dominated by a blocking high pressure system over Central and Western Europe, during the other months the "normal" situation with changing weather due to lows reaching Central Europe from the Atlantic returned. Remember that low clouds are assigned to this category only if they fill at least 5 to 6 oktas of the sky (see Sect. 3.1).

Figure 3 (bottom) displays the monthly frequency of occurrence of the "Contrails and cirrus", "All contrails" and "All cirrus" categories with respect to the time span where the cirrus level was visible from the ground. Of course, values are larger than in Fig. 3 (top). The "All contrails" line varies from $11 \%$ in July to $31 \%$ in May, and averages to $21 \%$. The "Contrails and cirrus" category follows quite closely the previous one at a somewhat lower level, while the "All cirrus" line ranges between $26 \%$ in July and $45 \%$ in May and June, with its average at $35 \%$. The fluctuations of these cloud categories are presumably due to changes of the large scale weather pattern, but their apparent positive correlation sug-

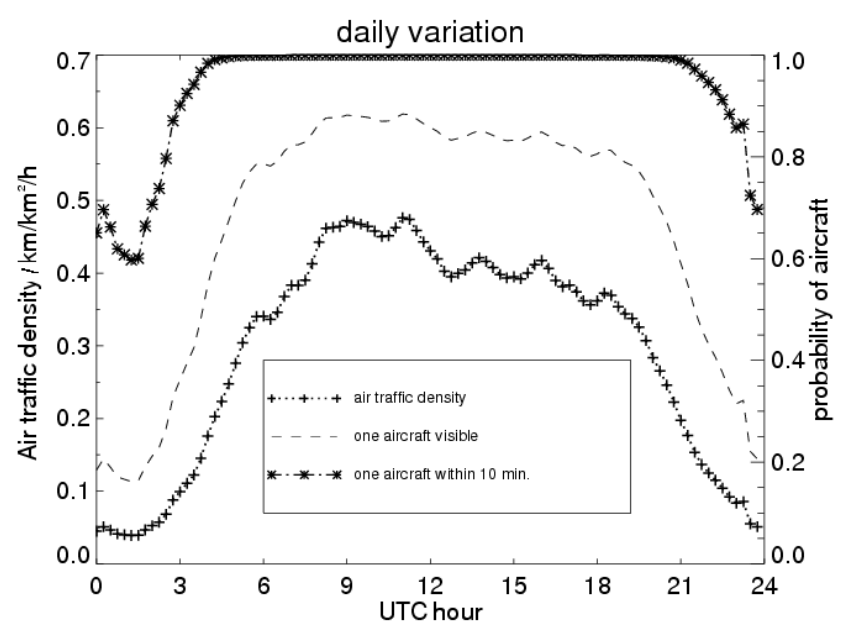

Fig. 5. Daily variation of higher level air traffic density (flown distance per square $\mathrm{km}$ and hour) in southern Bavaria according to data from EUROCONTROL for the summer schedule of 2004 $(+)$; probability that at least one aircraft is within Wolkam's field of view (dashed line); probability that at least one aircraft is visible in Wolkam's field of view within $10 \mathrm{~min}(*)$.

gests that when the conditions for cirrus formation and persistence are given (supersaturated air parcels) this also affects contrail formation. Compared to Wylie et al. (2005, Fig. 4) a reasonable agreement is found. They derived the monthly frequency of high clouds (above $6 \mathrm{~km}$ ) in the $20-60^{\circ} \mathrm{N}$ latitude belt from HIRS (High Resolution Infrared Radiation Sounder) aboard the NOAA satellites. It lies between 25 and $35 \%$ in the early 2000s. Due to the HIRS lower sensitivity to thin cirrus, these values are smaller than those derived from the SAGE (Stratospheric Aerosol and Gas Experiment) II limb sounder (Wylie and Wang, 1997), while they are higher than those obtained from ISCCP (Jin et al., 1996). The TOVS Path-B dataset (Scott et al., 1999) yields frequencies of cirrus clouds in northern midlatitudes of about $25 \%$ instead, and Hahn and Warren (2007) mention a frequency of high clouds over land of 45\% from surface observations between 1971 and 1996. Ground measurements at Hohenpeissenberg, Germany (some $50 \mathrm{~km}$ from DLR Oberpfaffenhofen) show cirrus frequencies between 40 and $70 \%$ in the late 1990s as a function of month (Trepte and Winkler, 2001).

\subsection{Diurnal cycle of cirrus and contrail occurrence}

Because of the restriction to daylight and the nonconsideration of hours with a sampling error exceeding 5\%, the diurnal cycle is investigated between 04:00 UTC and 19:00 UTC. Each time label in the figures in this section and in the next ones stands for the full following hour (e.g. the 04:00 UTC ocurrence represents the occurrence between 04:00 and 05:00 UTC). 
Figure 4 (top) depicts fractions of time of the whole measurement period. The frequencies of occurrence of the category "Low- and medium-level clouds" first decline from $56 \%$ (04:00-05:00 UTC) to 37\% (14:00-15:00 UTC) then slightly increase to $45 \%$ (18:00-19:00 UTC) over the course of the day. Surprisingly, the onset of (cumulus) convection due to increasing solar insolation is not observable in the dataset. On the one hand, this is due to the very definition of category 1 "Low- and medium-level clouds" where at least 5 to 6 oktas of the sky must be covered by clouds. On the other hand, the effect of convection on this cloud class is masked by other processes like the dissipation of low stratiform clouds with increasing solar insolation during the course of the day. The contrail occurrence does not show a significant diurnal cycle. It fluctuates between $8 \%$ and $14 \%$. This agrees well with the fact that air traffic density over Central Europe is very low at night, increases very rapidly between 02:00-03:00 UTC and 07:00-08:00 UTC, and then remains stable until 21:00 UTC when it decreases again (Fig. 5). In contrast, a considerable diurnal cycle for cirrus clouds exists: "All cirrus" frequency increases almost continuously from 11\% (05:00-06:00 UTC) to $29 \%$ (17:00-18:00 UTC). The "All ice clouds" class behaves similarly, changing from $16 \%$ in the morning to $31 \%$ in the afternoon. The frequencies relative to the period with visible cirrus level are displayed in Fig. 4 (bottom). Again the contrails do not show a diurnal cycle, their frequency of occurrence oscillates between $16 \%$ and $26 \%$. The cirrus clouds reveal a significant diurnal cycle: their frequencies rise from 23\% (05:00-06:00 UTC) to $48 \%$ (17:00-18:00 UTC). After an increase by $10 \%$ to $30 \%$ in the first morning hours, the "All ice clouds" occurrence remains more or less stable until 14:00-15:00 UTC and eventually increases again by $10 \%$ to $50 \%$ (17:00-18:00 UTC).

The cirrus cloud increase could be caused by natural or anthropogenic cirrus cloud formation mechanisms or by a combination of both. The main natural source of cirrus clouds during the spring and summer season is convection that is usually initiated in the afternoon. The main anthropogenic source of cirrus clouds, air traffic, affects ice cloud formation through contrails: during the course of the day more and more contrails could evolve into cirrus clouds, while during nighttime this cirrus would eventually have the time to dissipate while few contrails are produced due to the very small air traffic density (Fig. 5). Since it is not possible to quantify the amount of cirrus clouds produced by convection, we consider air traffic density Dtr shown in Fig. 5 to estimate the probability that at least one aircraft is within the Wolkam visible range. We assume a mean aircraft speed $V$ of $850 \mathrm{~km} / \mathrm{h}$, a visible area with a radius $R$ of $35 \mathrm{~km}$ and a Poisson distribution (rare events) for the probabilities that $N$ aircrafts are within this area. Then, the probability $P 0$ that no aircraft is visible reads $P 0=\exp \left(-D t r \cdot \pi \cdot R^{2} / V\right)$ and the probability that at least one aircraft is visible $P 1=1-P 0$. This probability does not change much during daytime (Fig. 5) and follows the curve of air traffic density. An aircraft crosses

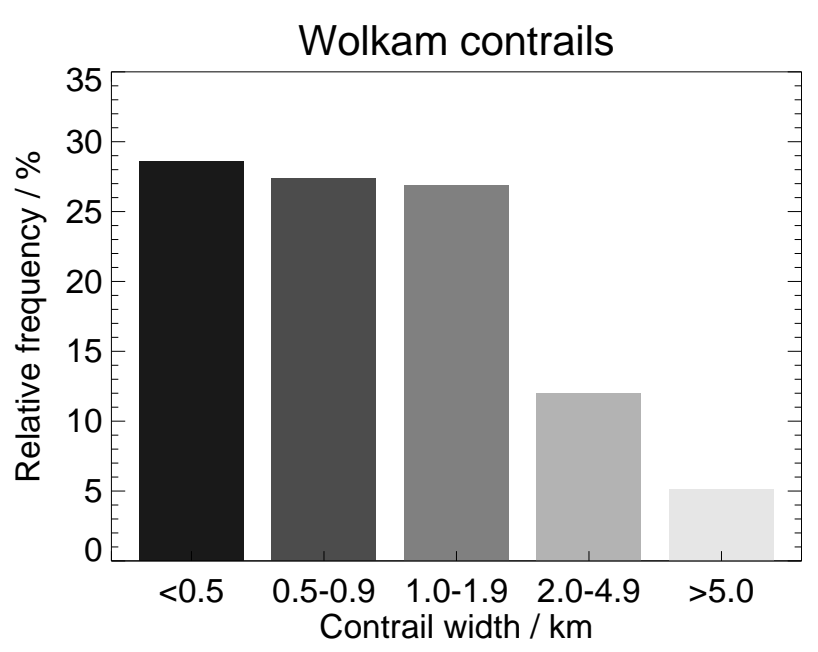

Fig. 6. Width distribution of 175 contrails observed using the allsky camera.

the area in approx. $4 \mathrm{~min}$, which defines the auto-correlation time scale. Thus, the probability that an aircraft crosses the Wolkam field of view within $10 \mathrm{~min}$ is constantly equal to unity between 04:00 and 21:00 UTC. We can first conclude that the weather situation, i.e. the probability of ice supersaturation at the flight levels, and not the air traffic density determines (i.e. limits) the occurrence of contrails at daytime over southern Bavaria. Secondly, from the observation that the occurrence of at least 3 min old contrails (see Sect. 3.1) is more or less constant over the day and from the constancy of the probability of seeing an airplane we can conclude that the probability of ice supersaturation at the flight levels does not significantly change during the day. However, implications about the vertical extension of such supersaturated regions cannot be drawn since air traffic mainly takes place in a very limited number of flight levels. Thus, the onset of convection in the afternoon could lead to more frequent cirrus formation and enhanced cirrus occurrence in (mainly lower) atmospheric levels during the course of the day. The strong daytime increase of cirrus occurrence in Wolkam images is also accompanied by an increase in cirrus cover as shown in Fig. 8. A second explanation for this increase might be the advection of contrail cirrus initiated hours before.

\subsection{Contrail width}

The contrail sample used to investigate contrail width consists of all $96+79$ contrails found in Wolkam data as illustrated in Sect. 3.2 and 3.3. It was divided into five width classes according to Fig. 6 and Sect. 3.4.

Figure 6 reveals that contrails of the categories $<0.5 \mathrm{~km}$ (29\%), $0.5-0.9 \mathrm{~km}$ and $1.0-1.9 \mathrm{~km}(27 \%$ each) occur almost equally frequent, their fraction diminishes considerably towards bigger widths, just $5 \%$ are more than $5 \mathrm{~km}$ wide 
Table 2. AVHRR/3 (NOAA18) and SEVIRI channel numbers and effective channel central wavelengths $\lambda_{\mathrm{c}}$.

\begin{tabular}{lrrrrrrrrrrrrr}
\hline \multicolumn{11}{c}{ AVHRR/3 } \\
\hline Channel & 1 & 2 & 3A & 3B & & & & 4 & 5 & \\
$\lambda_{\mathrm{c}} / \mathrm{\mu m}$ & 0.63 & 0.85 & 1.61 & 3.76 & & & & 10.77 & 12.00 & \\
\hline & & \multicolumn{1}{c}{ SEVIRI } & & & & & \\
\hline Channel & 1 & 2 & 3 & 4 & 5 & 6 & 7 & 8 & 9 & 10 & 11 & 12 \\
$\lambda_{\mathrm{c}} / \mu \mathrm{m}$ & 0.64 & 0.81 & 1.64 & 3.92 & 6.25 & 7.35 & 8.70 & 9.66 & 10.8 & 12.0 & 13.4 & 0.75 \\
\hline
\end{tabular}

Table 3. Comparison of contrail detection in Wolkam and NOAA/AVHRR data as a function of contrail width.

\begin{tabular}{lrrrrrr}
\hline Width/km & $<0.5$ & $0.5-0.9$ & $1.0-1.9$ & $2.0-4.9$ & $\geq 5.0$ & total \\
\hline Wolkam & 21 & 21 & 33 & 14 & 7 & 96 \\
Channel 5 & 0 & 5 & 19 & 9 & 5 & 38 \\
Temp. difference & 1 & 9 & 20 & 9 & 1 & 40 \\
Colour composite & 0 & 3 & 12 & 7 & 1 & 23 \\
CDA & 0 & 0 & 5 & 3 & 0 & 8 \\
\hline
\end{tabular}

(however, some of the broader contrails may have lost their linear shape and may have been classified as natural cirrus). The thinnest visible contrails feature a width of approximately $150 \mathrm{~m}$ ( 2 Wolkam pixels). This corroborates the suitability of the all-sky camera for observing contrails as stated in Sect. 3.2.

\section{Validation of AVHRR algorithms}

\subsection{Contrail detection efficiency}

Of the 96 contrails observed in Wolkam images at the time of a NOAA overpass, $38(40 \%)$ were identified in AVHRR channel 5 data, 40 (42\%) in temperature difference images and $23(24 \%)$ in the false colour composites; the CDA detected 8 contrails ( $8 \%$ ). A discrimination between the three different instruments on NOAA11, NOAA16 and NOAA17 is not possible due to the relatively small size of the sample.

Table 3 shows the detected contrails as a function of their width as determined from Wolkam data (see Sect. 4.3). The three visually interpreted image types (channel 5 temperature, temperature differences, false colour composite) exhibit an increasing detection efficiency as contrail width grows, but at large widths it decreases except in channel 5. Only one contrail narrower than $0.5 \mathrm{~km}$ was detectable by eye in an AVHRR temperature difference image. Although the number of contrails detected in brightness temperature and brightness temperature difference images is similar, the distribution of their width differs: their mean width is $2.6 \mathrm{~km}$ and $1.9 \mathrm{~km}$, respectively. On the one hand, this is explicable by the occurrence of the lowest temperatures (relative to the surround- ings) at the centre of wide contrails (Meyer et al., 2002), making them easily recognisable as dark lines. On the other hand, the smaller ice crystals of young and thus thin contrails in comparison to cirrus clouds lead to a larger difference in emissivity, which causes them to appear as bright lines in the temperature difference images while their temperature contrast with respect to the surroundings remains small. To ensure a high detection efficiency, both information source should be utilised, as it is done in the CDA. The colour composites feature a clearly lower detection efficiency than the first two image types: 23 detected contrails against 38 or 40 for brightness temperatures and brightness temperature differences plots respectively. The mean width of the contrails detected in false colour composites is $2.2 \mathrm{~km}$, and the width distribution is similar to the one detected with temperature difference images. Compared to the channel 5 temperature and the temperature difference plots, false colour composites contain information from thermal as well as from solar channels. Thin cirrus however is hardly visible in solar channels because solar channels introduce additional variability of the (relatively bright) background that hinders cirrus detection.

The CDA detects less than $10 \%$ of the contrails seen on Wolkam images, their width ranging from $1 \mathrm{~km}$ to $5 \mathrm{~km}$. Two of the eight contrails detected by the CDA could not be associated with single contrails, but only with a pair of them. These cases were counted once for the compilation of Table 3. This detection efficiency is significantly lower than the one specified in Mannstein et al. (1999), where a value of $30-50 \%$ was found in a sensitivity study against visually inspected AVHRR data. One reason for the lower detection efficiency compared to Wolkam is the inhomogeneous temperature distribution in the proximity of the all-sky camera 
location mainly caused by the urban heat island of Munich and the big lakes in the surrounding area (Lake Ammersee, Lake Starnberg). This aspect has already been investigated in Mannstein et al. (1999, Fig. 16), where the dependency of contrail cover on AVHRR channel 5 brightness temperatures inhomogeneity is estimated in order to correct CDA results in a statistical sense. In Mannstein et al. (1999, Fig. 15) the spatial distribution of AVHRR channel 5 brightness temperatures standard deviations is shown and confirms that the area investigated in this paper is particularly sensitive to this issue. However, in the present study no statistical correction in the sense of Mannstein et al. (1999) is possible. Furthermore, Mannstein et al. (1999) determined detection efficiency by comparison of CDA results with contrails manually detected in AVHRR images by some human observer. In order to reproduce these conditions, we can determine the CDA detection efficiency by comparing CDA results to the other AVHRR image types used for contrail detection (channel 4 minus channel 5 brightness temperature differences, channel 5 brightness temperatures and colour composites). Then, the CDA efficiency ranges from 20 to $35 \%$, which does not largely differ from the values contained in Mannstein et al. (1999).

Finally, we consider only those contrails whose width is at least comparable to the nominal AVHRR pixel size of $1.1 \mathrm{~km}$ at nadir that are expected to be detected by the four methods (temperatures, temperature differences, false colour composites, and CDA), according to the explanations given above. For temperature images and false colour composites we regard all 54 contrails with a width larger than $1 \mathrm{~km}$ : the resulting detection efficiencies amount to 61 and $37 \%$ respectively. For the temperature differences and the CDA a new reference ensemble of 47 contrails of width $1-5 \mathrm{~km}$ is built: the resulting detection efficiencies amount to 62 and $17 \%$ respectively. The fact that some wide contrails are not detected visually or by the CDA is probably related to their small optical thickness.

This analysis is not only interesting because it enables to evaluate the performance of the automated CDA algorithm. It also allows to better rate investigations of contrail occurrence based on visual evaluation of AVHRR data. In particular, the pioneering work of Bakan et al. (1994) is often cited as an early source of contrail occurrence (and coverage) information and has been used to calibrate numerical model analyses of global linear contrail coverage (see for instance Sausen et al., 1998). Bakan et al. (1994) is based on visual inspection of printed AVHRR brightness temperature images. Due to the reduced spatial resolution of their images of $20 \mathrm{~km}$ per mm, Bakan et al. (1994) assert that they can only detect older (thus wide) contrails, but they cannot quantify how many contrails they miss. Our investigation shows that thin narrow contrails represent the majority of the contrails observed with Wolkam and that AVHRR channel 5 temperatures enable to detect only a moderate fraction of these contrails. In fact, at least in our case, contrails smaller
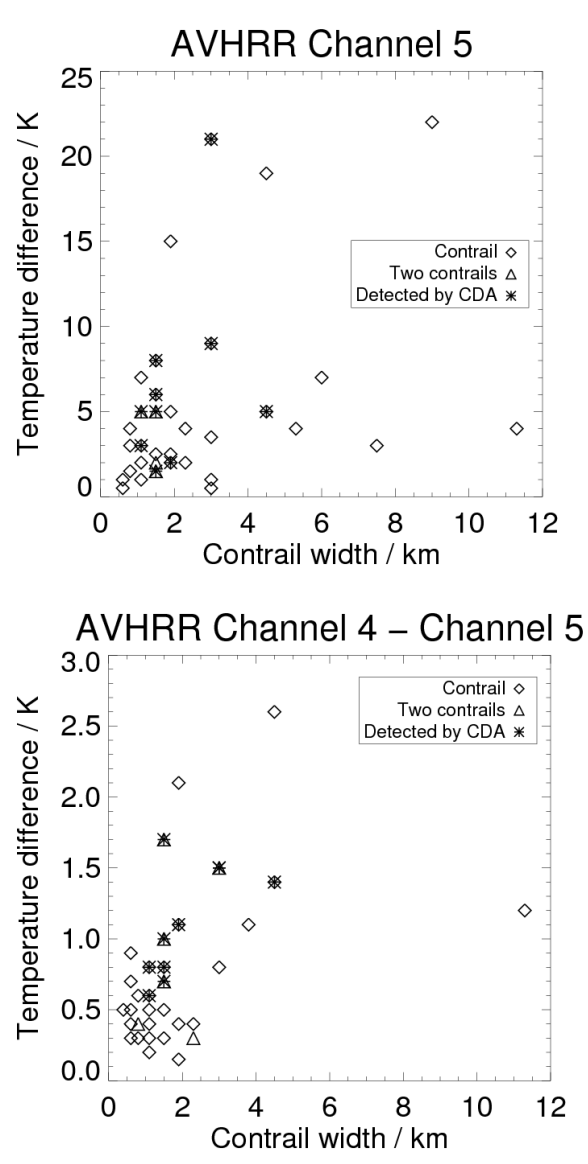

Fig. 7. Relationship between contrails and their surroundings derived from AVHRR data as a function of contrail width as obtained from Wolkam data. Top: Relationship of contrail width and brightness temperature difference between the contrail and its surroundings in AVHRR channel 5. Bottom: Relationship of contrail width and brightness temperature difference between the contrail and its surroundings in the temperature difference image (channel 4-5). In both graphics diamonds $(\diamond)$ mark single contrails detected in AVHRR data, triangles $(\triangle)$ indicate 2 contrails each, an asterisk $(*)$ at the centre marks the successful detection of the contrail by the CDA.

than $2 \mathrm{~km}$ make up $78 \%$ of the detected contrails and channel 5 temperature images show $32 \%$ of them. Furthermore, of the larger contrails ( $\geq 2 \mathrm{~km}), 67 \%$ are detected in channel 5 brightness temperatures. Of course, the surroundings of the Wolkam camera is particularly unfavourable with respect to contrail detection because of the inhomogeneous temperature distribution (see above). However, the concentration to this small spot makes a more detailed inspection possible than the full AVHRR swath. Using our figures, i.e. assuming that 1) Bakan et al. (1994) could observe only $67 \%$ of the large contrails $(\geq 2 \mathrm{~km})$ and no small contrail $(<2 \mathrm{~km})$, 2) large contrails ( $\geq 2 \mathrm{~km}$ ) represent only $32 \%$ of the contrails in the atmosphere, one could estimate detection efficiency in Bakan et al. (1994) to be approximately $21 \%$ ( $\approx 67 \%$ times 
$32 \%$ ). The question how this affects the values of contrail occurrence or contrail coverage quantitatively is even more difficult to assess. Nonetheless, it is evident that only a relatively small fraction of the existing contrails could be identified by Bakan et al. (1994).

\subsection{Relationship of contrail width and brightness tem- perature change}

The brightness temperature differences between each detected contrail and its surroundings are plotted in Fig. 7 (top) for channel 5 temperatures and for brightness temperature differences (bottom).

Regarding channel 5 temperatures, the minimum difference for a detectable contrail is $1-2 \mathrm{~K}$, most differences are smaller than $10 \mathrm{~K}$, the average is $5.2 \mathrm{~K}$. A positive correlation between width and temperature difference is identifiable. That means that the lowest temperatures occur in the centre of wide contrails, consistent with the findings of Meyer et al. (2002). With the AVHRR resolution of about $1 \mathrm{~km}$, there is the chance that a contrail does not fill a satellite pixel completely, especially for narrow contrails $(<2 \mathrm{~km})$. Thus, contrail brightness temperatures are contaminated by their surrounding temperatures such that temperature differences are smaller than expected. The mean difference of $5.2 \mathrm{~K}$ also matches the $5.4 \mathrm{~K}$ established by Meyer et al. (2002).

Considering the temperature difference between channel 4 and 5 (Fig. 7 (bottom), symbols as before), the minimum contrast value between contrail and surroundings is $0.2-$ $0.3 \mathrm{~K}$, the largest values are $2-3 \mathrm{~K}$, the average is $0.8 \mathrm{~K}$. The mentioned positive correlation is more pronounced in this case: in accordance with Meyer et al. (2002) the temperature difference increases towards the centre of wide contrails. Again, partly covered pixels induce smaller temperature differences and this is probably the case for narrow contrails.

\section{Validation of SEVIRI algorithms}

\subsection{Contrail detection efficiency}

Of the 79 contrails identified on the all-sky camera images (Sect. 3.3), 12 (15\%) were identifiable in the SEVIRI temperature difference images and 7 (9\%) in the colour composites. For a few points in time the comparison of Wolkam images with the false colour composites was not possible due to the low sun elevation. The CDA adapted to SEVIRI detected 7 contrails (9\%). Comparing the automated contrail detection with the best visual inspection (temperature difference images) yields a detection efficiency of $58 \%$. These results are shown in Table 4 as a function of contrail width.

The detection efficiency in brightness temperature differences and false colour images of 9 and $15 \%$ is significantly lower than that of the comparable AVHRR visual tools that amounts to $42 \%$ and $24 \%$ respectively; the CDA efficiency is of equal magnitude (9\% for SEVIRI against $8 \%$ for AVHRR). Only the CDA detection efficiency when determined against the number of contrails detected in brightness temperature difference images is higher and almost reaches $60 \%$. The analysis of contrail widths was not executed due to the low number of contrails (79) observed in SEVIRI images. The low efficiency of the visual methods applied to SEVIRI data is caused by the low resolution of $3 \mathrm{~km} / \mathrm{pixel}$ for SEVIRI infrared channels in contrast to $1.1 \mathrm{~km} /$ pixel for AVHRR. This difference is enhanced by SEVIRI's slant view on Central Europe. It has to be noticed that the majority of the investigated contrails has a width smaller than SEVIRI's spatial resolution. Nonetheless, even contrails with widths smaller than $1 \mathrm{~km}$ were sporadically visible or detected by the CDA.

In the 2797 points in time processed during May 2007, the CDA detected contrail pixels in 299 cases, i.e. $11 \%$ of the time. The occurrence for Wolkam data is $31 \%$, nearly three times as high. However this percentage cannot be directly compared to the MSG/SEVIRI values because only daylight hours and times with a visible cirrus level could be used. The frequency averaged over full days should be somewhat lower due to lower nocturnal contrail coverage (Mannstein et al., 1999). The remaining difference is probably caused by thin contrails which cannot be detected in SEVIRI imagery. At 103 of the mentioned 299 points in time the cirrus level was visible in Wolkam images making the comparison of the CDA results to ground-based imagery possible. In 28 cases $(27 \%)$ the contrails marked by the CDA were identical to contrails photographed by the all-sky camera. In most of the remaining cases the CDA marked elongated, some $10 \mathrm{~km}$ wide cirrus streaks that are not identified as contrails in the visual interpretation of the Wolkam images. Some of them might have been induced by air traffic, but are in a later stage of their evolution into contrail cirrus. This kind of clouds is known to be responsible for most misdetections generated by the AVHRR version of the CDA (Mannstein et al., 1999).

\subsection{Validation of MeCiDA cirrus diurnal cycles: April- September 2007}

The analysis of the distribution of cirrus occurrence during the day obtained by MeCiDA from April to September 2007 over the Wolkam location as described in Sect. 3.3 yields a slight diurnal cycle featuring a 69\% minimum at 09:00 UTC and a 76\% maximum at 21:00 UTC. Between these times there is a gentle increase during daytime and an almost as gentle decrease during nighttime, both of them are not monotone (Fig. 8).

The Wolkam camera enabled to analyse the diurnal cycle of ice clouds between 04:00 UTC (04:00-05:00 UTC) and 18:00 UTC (18:00-19:00 UTC). In Fig. 8 the occurrence of the "All ice clouds" is plotted because it is rather comparable to satellite data that cannot distinguish between contrails and cirrus. A rise of the cirrus frequency from $30 \%$ at 04:00 UTC to $51 \%$ at 17:00 UTC is visible. Both diurnal 
Table 4. Comparison of contrail detection in Wolkam and MSG/SEVIRI data as a function of their width.

\begin{tabular}{lrrrrrr}
\hline Width $(\mathrm{km})$ & $<0.5$ & $0.5-0.9$ & $1.0-1.9$ & $2.0-4.9$ & $\geq 5.0$ & total \\
\hline Wolkam & 29 & 27 & 14 & 7 & 2 & 79 \\
Temp. difference & 0 & 2 & 6 & 4 & 0 & 12 \\
Colour composite & 0 & 0 & 1 & 4 & 2 & 7 \\
CDA & 0 & 1 & 2 & 2 & 2 & 7 \\
\hline
\end{tabular}

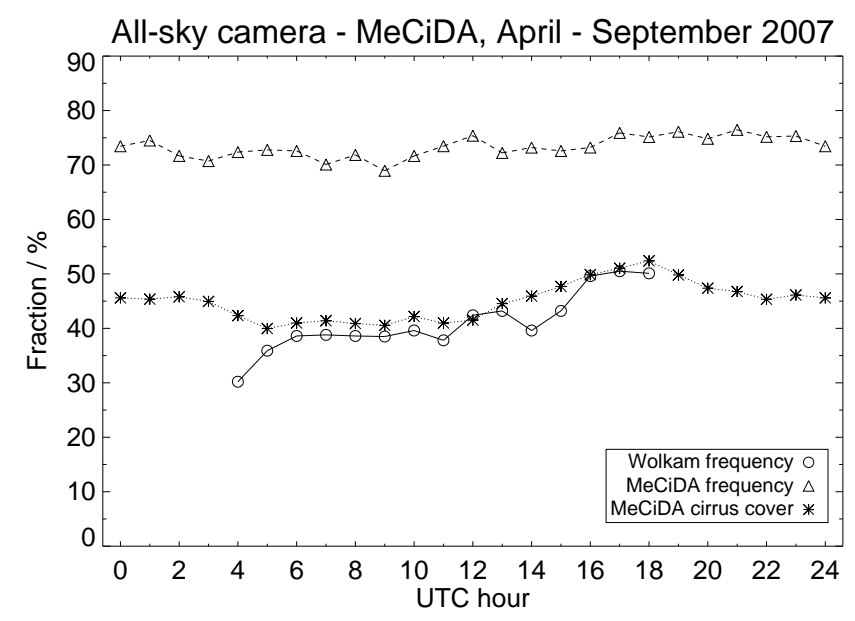

Fig. 8. Diurnal cycles of the frequency of "All ice clouds" occurrence obtained from all-sky camera data $(\bigcirc)$ and via the MeCiDA algorithm $(\triangle)$ and the diurnal cycle of cirrus cloud coverage gained from $\operatorname{MeCiDA}(*)$.

cycles have in common that this frequency is larger in the evening than in the morning hours, but the daytime increase gained from Wolkam is remarkably more pronounced. The fact that the values obtained by MeCiDA are considerably higher has various reasons: 1) MeCiDA does not only detect (thin) cirrus but also icy tops of high reaching convective clouds which Wolkam classifies as "Low- and mediumlevel clouds"; 2) the classification of cloud type in the poorly observable parts of the camera images close to the horizon is difficult, especially when a low cloud above the camera location is present; 3) the region observed with SEVIRI is somewhat larger and the probability of cirrus occurrence increases; 4) MeCiDA seems to often classify single isolated pixels as cloudy although no large scale cirrus pattern is visible in the surroundings.

The diurnal cycle of MeCiDA cirrus cloud coverage features a 40\% minimum at 05:00 UTC and a 52\% maximum at 18:00 UTC, in between there is a largely monotone rise respectively descent (Fig. 8). The coincidence of the absolute values (almost always closer than $5 \%$ ) of the diurnal cycle of MeCiDA cirrus cloud coverage and the diurnal cycle of Wolkam "All ice clouds" occurrence is incidental, but the common trend of both curves, i.e. their increase during the day, is related: When cirrus cover increases, its probability of occurrence inside the all-sky camera field of view increases also. This feature is also observed in the month of July 2007 (and probably in other months as well).

A similar behaviour is shown in Stubenrauch et al. (2006): From the TOVS Path-B dataset they extracted diurnal cycles of high cloud amount in summer over northern midlatitudes land showing increases from $30 \%$ in the morning to $40 \%$ in the afternoon.

At times of low sun elevation the sky in Wolkam data appears more palish than otherwise and cirrus detection is more difficult. However, the reduced visibility of cirrus clouds at these times obviously does not induce a systematic error because the difference of the two graphs depicting relative frequencies in Fig. 8 are not symmetric with respect to noontime (11:00 UTC). The improved visibility of thin cirrus before sunrise and after sunset is also noticeable in Wolkam images, but this effect has no influence on the analysis as the period between sunset and sunrise is not considered in the data evaluation.

\section{Conclusions and outlook}

In contrast to satellite observations all-sky cameras feature high spatial and temporal resolution, thus nearly all contrails can be detected by visual inspection in a limited area around the camera location and an accurate cloud classification can be performed. Thus, they qualify as excellent instruments for the validation of satellite cloud algorithms.

In this study we concentrated on contrails and cirrus clouds, in particular on the validation of the contrail detection algorithm (CDA) by Mannstein et al. (1999), applied to both NOAA/AVHRR and MSG/SEVIRI, and on the validation of the MeCiDA cirrus cloud detection scheme (Krebs et al., 2007) applied on MSG/SEVIRI. This analysis was complemented by the visual inspection of various satellite channels and channel combinations: infrared window channel brightness temperatures, infrared window channel brightness temperature differences and false colour composites incorporating both solar and thermal information. This additional investigation enabled us to better classify the scores of the automated algorithms and built a bridge to the likewise visual inspection of the ground based Wolkam dataset. A summary of the datasets used is given in Table 1 . 
The analysis of the six months Wolkam time series (AprilSeptember 2007) revealed that contrails can be seen from ground $12 \%$ of the time, cirrus clouds $20 \%$. The frequency with respect to the period with visible cirrus level are $21 \%$ for contrails and $35 \%$ for cirrus, with considerable variations between the months. The frequency of contrail occurrence does not vary significantly during daytime. In contrast, cirrus clouds show a distinct diurnal cycle: their relative frequency of occurrence namely rises from $27 \%$ to $48 \%$ during daylight hours with respect to the time span characterised by visible cirrus level. The comparison of this frequency to the one gained by MeCiDA data indicates that MeCiDA values are notedly higher at all hours of the day. However, when looking at cirrus cloud cover from MeCiDA, a similar increase is found. This could be a hint that contrails with a lifetime of some hours evolve into cirrus clouds and cause the diurnal increase observed, but is more likely caused by convection.

Around $42 \%$ of the contrails identified in Wolkam images at the times of NOAA/AVHRR satellite overpasses could be recognised in channel 5 temperature and channel 4 minus channel 5 temperature difference images, only $24 \%$ were identifiable in AVHRR colour composites, the CDA detected $8 \%$. The percentage of contrails detected in AVHRR images increases with their width, but decreases again once widths are larger than $5 \mathrm{~km}$, except for channel 5 temperatures. Regarding the contrails with widths of 1 to $5 \mathrm{~km}$, detection efficiency of temperature and temperature difference images is $60-65 \%$. The detection efficiency of the CDA is $17 \%$. In order to compare with the detection efficiency (30-50\%) from Mannstein et al. (1999), one must juxtapose visually detected AVHRR contrails and CDA contrails. In this case, the CDA detects approx. $28 \%$ of the contrails.

According to our findings the early model studies, which refer to Bakan et al. (1994), should have missed $80 \%$ of the linear contrails visible from the ground. But the fact that the large scale "contrail areas" considered in Bakan et al. (1994) cannot be judged from ground observations as they are formed mainly by aged contrails does not allow to draw the conclusion that the coverage by linear contrails was underestimated by this factor. Furthermore, our results show that the analyses by Mannstein et al. (1999) and Meyer et al. (2002) have underestimated the coverage by linear contrails by a factor of - at least - two despite of the statistical correction applied to the data. This estimate is based on the fact that the CDA detection efficiency amounts to $8 \%$ compared to all contrails visible from the ground and to $20-35 \%$ compared to those contrails that have a noticeable impact on the radiation field and are therefore also detectable by eye in the AVHRR data.

As far as contrail detection in MSG/SEVIRI is concerned, 79 contrails were visible, $15 \%$ of these could be identified in temperature difference images, $10 \%$ in the colour composites, the CDA detected $9 \%$. This shows a worse detection than with AVHRR, due to the coarser spatial resolution. During May 2007 the CDA yielded a 11\% contrail frequency within the area observed by the Wolkam camera. During the periods of visible cirrus level, Wolkam images confirmed $27 \%$ of the CDA contrails and showed a high false alarm rate of the SEVIRI version of the CDA, mainly produced by elongated cirrus structures.

To achieve a substantially improved estimation of the climatic impact of contrails, an upgraded all-sky camera system allowing for an automated image interpretation is necessary. With the current system automated analysis was not feasible due to reflections, blurring and a large area affected by blooming caused by the sun. Besides a sensor with higher resolution the new system should feature a device to block direct sunlight from the dome. Furthermore it is important that the new camera system is able to operate during nighttime (such a system is described in Seiz et al., 2007). In addition the acrylic dome should be heated to obtain time series without interruptions due to dew and rime. The utilisation of a camera system which is calibrated with respect to brightness temperature differences may enable the estimation of optical thickness by using the contrast between contrail and surrounding sky. The employment of two all-sky cameras would allow for the calculation of contrail height. Although, despite of all mentioned improvements, the creation of complete time series will not be possible due to the occurrence of clouds below the cirrus level, these improvements would offer the possibility to further the understanding of the contrailinduced climatic effects by exploiting the full potential of allsky cameras.

Acknowledgements. We thank EUROCONTROL and Kaspar Graf (DLR) for the provision of air traffic data, and Simon Unterstrasser for careful reading of the manuscript and valuable comments.

Edited by: A. A. Kokhanovsky

\section{References}

Appleman, H.: The formation of exhaust contrails by jet aircraft, B. Am. Meteorol. Soc., 34, 14-20, 1953.

Bakan, S., Betancor, M., Gayler, V., and Grassl, H.: Contrail frequency over Europe from NOAA-satellite images, Ann. Geophys., 12, 962-968, 1994, http://www.ann-geophys.net/12/962/1994/.

Fichter, C., Marquart, S., Sausen, R., and Lee, D.: The impact of cruise altitude on contrails and related radiative forcing, Meteorol. Z., 14(4), 563-572, 2005.

Forster, P., Ramaswamy, V., Artaxo, P., Berntsen, T., Betts, R., Fahey, D., Haywood, J., Lean, J., Lowe, D., Myhre, G., Nganga, J., Prinn, R., Raga, G., Schulz, M., and Van Dorland, R.: Changes in atmospheric constituents and in radiative forcing, in: Climate change 2007: The physical science basis, Technical Report 2007, Intergovernmental Panel on Climate Change (IPCC), IPCC Secretariat, c/o World Meteorological Organization, Geneva, Switzerland, http://www.ipcc.ch, 2007.

Gierens, K. M., Kärcher, B., Mannstein, H., and Mayer, B.: Aerodynamic contrails: Phenomenology and flow physics, J. Atmos. Sci., 66, 217-226, doi:10.1175/2008JAS2767.1, 2009. 
Goodrum, G., Kidwell, K., and Winston, E. W.: NOAA-KLM user's guide, Technical report, NOAA/NESDIS, National Climatic Data Center, 151 Patton Ave., Asheville, NC 28801-5001, http://www2.ncdc.noaa.gov/docs/klm/index.htm, 2003.

Hahn, C. and Warren, S.: A gridded climatology of clouds over land (1971-96) and ocean (1954-97) from surface observations worldwide, Numeric Data Package NDP-026E ORNL/CDIAC153, CDIAC, Department of Energy, Oak Ridge, Tennessee, 2007.

Inoue, T.: On the temperature and effective emissivity determination of semi-transparent cirrus clouds by bi-spectral measurements in the $10 \mu \mathrm{m}$ window region, J. Meteorol. Soc. Jpn., 63(1), 88-99, 1985.

IPCC: Climate change 2007: The scientific basis, Technical report, Intergovernmental Panel on Climate Change (IPCC), IPCC Secretariat, c/o World Meteorological Organization, Geneva, Switzerland, 2007.

Jin, Y., Rossow, W., and Wylie, D.: Comparison of the climatologies of high-level clouds from HIRS and ISCCP, J. Climate, 9, 2850-2879, 1996.

Krebs, W., Mannstein, H., Bugliaro, L., and Mayer, B.: Technical note: A new day- and night-time Meteosat Second Generation Cirrus Detection Algorithm MeCiDA, Atmos. Chem. Phys., 7, 6145-6159, doi:10.5194/acp-7-6145-2007, 2007.

Kärcher, B., Mayer, B., Gierens, K., Burkhardt, U., Mannstein, H., and Chatterjee, R.: Aerodynamic contrails: Microphysics and optical properties, J. Atmos. Sci., 66, 227-243, doi:10.1175/2008JAS2768.1, 2009.

Lee, D., Fahey, D., Forster, P., Newton, P., Wit, R., Lim, L., Owen, B., and Sausen, R.: Aviation and global climate change in the 21st century, Atmos. Environ., 53, 3520-3537, 2009.

Mannstein, H., Meyer, R., and Wendling, P.: Operational detection of contrails from NOAA-AVHRR-data, Int. J. Rem. Sens., 20(8), 1641-1660, 1999.

Marquart, S.: Klimawirkung von Kondensstreifen: Untersuchungen mit einem globalen atmosphärischen Zirkulationsmodell, Dissertation, Fakultät für Physik der Ludwig-Maximilians-Universität München, 2003.

Meerkötter, R., Schumann, U., Doelling, D. R., Minnis, P., Nakajima, T., and Tsushima, Y.: Radiative forcing by contrails, Ann. Geophys., 17, 1080-1094, 1999, http://www.ann-geophys.net/17/1080/1999/.

Meyer, R., Mannstein, H., Meerkötter, R., Schumann, U., and Wendling, P.: Regional radiative forcing by line-shaped contrails derived from satellite data, J. Geophys. Res., 107(D10), 4104, doi:10.1029/2001JD000426, 2002.

Meyer, R., Buell, R., Leiter, C., Mannstein, H., Pechtl, S., Oki, T., and Wendling, P.: Contrail observations over Southern and Eastern Asia in NOAA/AVHRR data and comparisons to contrail simulations in a GCM, Int. J. Rem. Sens., 28(9), 2049-2069, 2007.
Minnis, P., Young, D., Garber, D., Nguyen, L., Smith Jr., W., and Palikonda, R.: Transformation of contrails into cirrus during SUCCESS, Geophys. Res. Lett., 25, 1157-1160, 1998.

Minnis, P., Ayers, J., Palikonda, R., and Phan, D.: Contrails, cirrus trends, and climate, J. Climate, 17, 1671-1685, 2004.

Minnis, P., Palikonda, R., Walter, B. J., Ayers, J. K., and Mannstein, H.: Contrail properties over the eastern North Pacific from AVHRR data, Meteorol. Z., 14(4), 515-523, doi:10.1127/09412948/2005/0056, 2005.

Sassen, K.: Contrail-cirrus and their potential for regional climate change, B. Am. Meteorol. Soc., 78, 1885-1903, 1997.

Sausen, R., Gierens, K., Ponater, M., and Schumann, U.: A diagnostic study of the global distribution of contrails: Part I: Present day climate, Theor. Appl. Climatol., 61, 127-141, 1998.

Schmetz, J., Pili, P., Tjemkes, S., Just, D., Kerkmann, J., Rota, S., and Ratier, A.: An introduction to Meteosat Second Generation (MSG), B. Am. Meteorol. Soc., 83(7), 977-992, 2002.

Schmidt, E.: Die Entstehung von Eisnebel aus den Auspuffgasen von Flugmotoren, Schriften der Deutschen Akademie der Luftfahrtforschung, 44, 1-15, 1941.

Schumann, U.: On conditions for contrail formation from aircraft exhausts, Meteorol. Z., 5, 4-23, 1996.

Schumann, U.: Formation, Properties and climate effects of contrails , C. R. Phys., 6, 549-565, 2005.

Scott, N., Chèdin, A., Armante, R., Francis, J., Stubenrauch, C. J., Chaboureau, J.-P., Chevallier, F., Claud, C., and Chèruy, F.: Characteristics of the TOVS Pathfinder Path-B dataset, B. Am. Meteorol. Soc., 80, 2679-2701, 1999.

Seiz, G., Shields, J., Feister, U., Baltsavias, E., and Gruen, A.: Cloud mapping with ground-based photogrammetric cameras, Int. J. Rem. Sens., 28(9), 2001-2032, 2007.

Stordal, F., Myhre, G., Stordal, E. J. G., Rossow, W. B., Lee, D. S., Arlander, D. W., and Svendby, T.: Is there a trend in cirrus cloud cover due to aircraft traffic?, Atmos. Chem. Phys., 5, 2155-2162, doi:10.5194/acp-5-2155-2005, 2005.

Stubenrauch, C., Chèdin, A., Rädel, G., Scott, N., and Serrar, S.: Cloud properties and their seasonal and diurnal variability from TOVS Path-B, J. Climate, 19, 5531-5553, 2006.

Stuber, N. and Forster, P.: The impact of diurnal variations of air traffic on contrail radiative forcing, Atmos. Chem. Phys., 7, 3153-3162, doi:10.5194/acp-7-3153-2007, 2007.

Trepte, S. and Winkler, P.: Langfristige meteorologische Veränderungen und UV-Strahlung, Ozonbulletin 81, Deutscher Wetterdienst, Offenbach am Main, 2001.

Wylie, D. and Wang, P.: Comparison of cloud frequency data from HIRS and SAGE II, J. Geophys. Res., 102, 29893-29900, 1997.

Wylie, D., Jackson, D., Menzel, W., and Bates, J.: Trends in global cloud cover in two decades of HIRS observations, J. Climate, 18, 3021-3031, 2005.

Zerefos, C. S., Eleftheratos, K., Balis, D. S., Zanis, P., Tselioudis, G., and Meleti, C.: Evidence of impact of aviation on cirrus cloud formation, Atmos. Chem. Phys., 3, 1633-1644, doi:10.5194/acp3-1633-2003, 2003. 